\title{
Time-Resolved Photoelectron Spectroscopy to Probe Ultrafast Charge Transfer and Electron Dynamics in Solid Surface Systems and at Metal- Molecule Interfaces
}

\author{
Luca Castiglioni*, Michael Greif, Dominik Leuenberger, Silvan Roth, Jürg Osterwalder, and \\ Matthias Hengsberger
}

\begin{abstract}
Photoelectron spectroscopy (PES) is a versatile tool, which provides insight into electronic structure and dynamics in condensed matter, surfaces, interfaces and molecules. The history of PES is briefly outlined and illustrated by current developments in the field of time-resolved PES. Our group's research is mostly aimed at studying ultrafast processes and associated lifetimes related to electronic excitation at solid surfaces.
\end{abstract}

Keywords: Electron transfer · Diamondoids · Molecular layers · Photoelectron spectroscopy · Surface dynamics · Time-resolved 2PPE

\section{Introduction}

Electronic processes at surfaces such as charge transfer are of highest importance in many chemical reactions, in heterogeneous catalysis, or in photovoltaic devices. Conventional photoelectron spectroscopy (PES $)^{[1]}$ has been the key tool to investigate the electronic structure of solid matter as well as single molecules and atoms for nearly half a century. The advent of femtosecond lasers in the 1990s paved the way for time-resolved experiments to probe electron dynamics as well as the motion of nuclei. We want to provide a short introduction to photoelectron spectroscopy and briefly discuss some recent studies of fundamental processes at surfaces by means of time-resolved PES. Furthermore we give an outlook on potential new experiments within the framework of the NCCR MUST.
${ }^{\star}$ Correspondence: Dr. L. Castiglioni Physik-Institut Universität Zürich

Winterthurerstrasse 190

$\mathrm{CH}-8057$ Zürich

Tel.: +41446356695

Fax: +4144635 5704

E-mail: luca.castiglioni@physik.uzh.ch

\section{Brief History of Photoelectron Spectroscopy}

The photoelectric effect, which was first described by Hertz ${ }^{[2]}$ in 1887 and then rationalized by Einstein ${ }^{[3]}$ invoking the quantum nature of light, constitutes the underlying mechanism of photoelectron spectroscopy. Einstein stated that the energy of the emitted electron would be given by

$$
E_{k i n}=h v-\phi
$$

with $h v$ being the energy of the absorbed photon and $\phi$ the work function, i.e. the minimum energy required to remove one electron from a solid into vacuum. It took yet another half a century of significant theoretical and technical breakthroughs until the photoelectric effect could be used as a spectroscopic tool. X-ray photoelectron spectroscopy (XPS) was developed in the 1950s and Siegbahn and coworkers ${ }^{[4,5]}$ were the first to produce high-resolution XPS spectra showing the core levels of the elements under investigation. Incorporating the binding energy, $E_{B}$, in Eqn. (1) directly shows how the core level positions can be calculated from the kinetic energy of the detected electrons:

$E_{k i n}=h v-\phi-E_{B}$

Siegbahn also noticed that his method is sensitive to the chemical environment of the ionized atom resulting in shifts of the measured binding energies, and he henceforth referred to it as electron spec- troscopy for chemical analysis (ESCA). At the same time Spicer and coworkers started measuring the band gaps and electron affinities in semiconductors using ultraviolet photoelectron spectroscopy (UPS). ${ }^{6]}$ The short inelastic mean free path of electrons at typical XPS energies (0.1-1.5 keV) inside matter of $\sim 1 \mathrm{~nm}$ makes photoelectron spectroscopy a highly surface-sensitive method. At typical EUV energies (20-100 $\mathrm{eV}$ ), the mean free path can be as short as one or two atomic distances. ${ }^{[7]} \mathrm{A}$ general energy scheme of a metal surface and different photoelectron emission processes is depicted in Fig. 1.

While conventional PES can only probe occupied levels, information about unoccupied levels is highly desirable for many reasons. Hot, photoexcited electrons play a vital role in a variety of processes at surfaces and interfaces such as chemical reactions and electron transport. ${ }^{[8]}$ One method to probe unoccupied levels is inverse photoemission spectroscopy (IPES). ${ }^{[9]}$ Electrons from a collimated beam with well-defined energy hit the surface and couple with high-lying unoccupied electronic states. Some of the subsequent decays to lower-lying unoccupied states are radiative and the emitted photons can be detected to generate an energy spectrum. PES and IPES are complementary techniques in the sense that PES probes states below the Fermi level $\left(E_{F}\right)$, whereas IPES can access unoccupied states above $E_{F}$. While PES delivers an electron removal spectrum, one can collect an electron addition spectrum with IPES. 


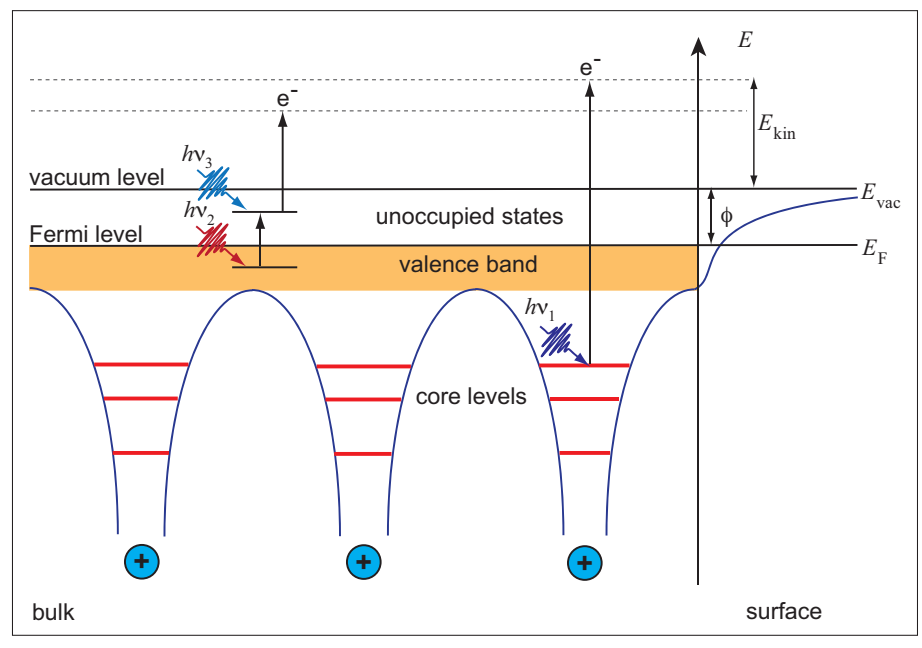

Fig. 1. Core and valence levels at a metal surface that can be probed by photoelectron spectroscopy. One $\mathrm{X}$-ray photon $\left(h v_{1}\right)$ causes emission of a core electron. Two UV-VIS photons $\left(h v_{2}\right.$ and $\left.h v_{3}\right)$ cause emission of a valence band electron via an intermediate state above the Fermi level.

\section{Time-resolved Photoelectron Spectroscopy}

Besides the energetics of unoccupied levels the temporal evolution of hot electron distributions is of most interest. Whereas upper limits of charge carrier relaxation times in metals can be estimated from the analysis of the linewidth of spectra obtained by conventional PES and related techniques, two-photon photoemission (2PPE) allows the dynamics of excited electrons to be studied directly in the time domain. ${ }^{[10]}$ The energy range of accessible unoccupied states between $E_{F}$ and $E_{v a c}$ is limited since the photon energy of both pump- and probe-pulses needs to be lower than $\phi$ to avoid direct photoemission.

Studying the dynamics of hot electrons at metal surfaces was one of the primary motivations for 2PPE experiments since excitation of adsorbed molecules by charge transfer appears to be one of the predominant mechanisms in surface photochemistry. ${ }^{[11]}$ The first surface that was extensively studied by 2PPE was $\mathrm{Cu}(100) .{ }^{[12,13]}$ Knoesel et al. determined relaxation times to vary from $250 \mathrm{fs}$ at 0.1 $\mathrm{eV}$ above $E_{F}$ and $20 \mathrm{fs}$ at $2 \mathrm{eV}$ as well as different lifetimes for excitation of $\mathrm{sp}$ - and d-band electrons. ${ }^{[14]}$

Another interesting feature to be studied by 2PPE are image potential states, which were first predicted in 1978 and which are well described by theory. ${ }^{[15]}$ They arise from an electron being trapped in front of the surface in the potential well generated by its own attractive image force and the band gap. They form Rydberg-like series of states with a main quantum number $n$ and $l=0$. Lifetimes around $30 \mathrm{fs}$ were measured on $\mathrm{Cu}(100)$ for the $n=1$ state $^{[16]}$ and it can be deduced from theory that the lifetime will scale with $n^{3}$. Höfer et al. managed to prepare image potential states with $n=4 . .66$ and measured lifetimes between 0.63 and 2.0 ps respectively. For coherent excitation of several states centered around $\tilde{n}=7$ they observed quantum beating with a period of $800 \mathrm{fs}$ resulting from the electron wave packet oscillating in front of the surface. ${ }^{[17]}$

More recently the availability of wellcharacterized ultra-short attosecond ( 1 as = $10^{-18} \mathrm{~s}$ ) laserpulsesopened the doortotime-resolved experiments of bound electrons. ${ }^{[18,19]}$ Photoemission is usually described as a three-step process: excitation, transport and escape of the photoelectron through the surface. ${ }^{[20]}$ In a proof-of-principle experiment, Cavalieri et al. delivered the first direct as-time-resolved measurement of electron transport in solid matter.[21] A 300 as XUV pulse was focused on a $\mathrm{W}(110)$ surface and the emitted photoelectrons were streaked in the phase-matched co-propagating NIR field. They were able to determine a relative delay in the emission of electrons from the conduction band and the $4 f$ core level of $\sim 100$ as and gave an estimate of the absolute delay between initial excitation and escape through the surface of $\sim 60$ and $\sim 150$ as, respectively. In 2010 the same group reported a time delay of $21 \pm 5$ as in the photoemission of electrons liberated from the $2 p$ orbitals in neon with respect to those released from the $2 s$ orbital. ${ }^{[22]}$ To our knowledge, this is currently the shortest timescale ever measured in an optical spectroscopy experiment.

\section{Surface Physics at the University of Zurich}

Our group's activities started in the mid-1990s with the construction of an electron-spectrometer for angle-resolved photoemission (ARPES) experiments in the XPS and UPS regime. ${ }^{[23]}$ A monochromatized high-flux $\mathrm{He}$ discharge lamp in combination with a $2 \pi$ solid angle sample goniometer allows thermally populated valence states up to $5 k_{B} T$ above the Fermi level to be mapped. Systems that were studied in the following years include observation of the magnetic exchange splitting in nickel,[24] step-induced surface states on $\mathrm{Cu}(332),{ }^{[25]}$ the influence of $\mathrm{CO}$ adsorption on Shockley type surface states on $\mathrm{Cu}(111)^{[26]}$ or electron coherence in a melting $\mathrm{Pb}$ monolayer on $\mathrm{Cu}(111){ }^{[27]} \mathrm{In}$ addition to band mapping, the same apparatus can be used for photoelectron diffraction experiments in order to characterize the structure of the surface and adsorbate geometries. The absorption of oxygen on Rh(111) was studied by X-ray photoelectron diffraction (XPD) ${ }^{[28]}$ and the formation of subsurface oxygen could be directly observed.[29] Utilizing the same approach, the absolute orientation of chiral molecules can be determined as it was shown for heptahelicene, $\mathrm{C}_{30} \mathrm{H}_{18}$, on copper surfaces ${ }^{[30]}$ and tartaric acid on $\mathrm{Cu}(110) .{ }^{[31]}$ The group also established and operates the COPHEE (complete photoemission experiment) endstation at the Swiss Light Source (SLS) ${ }^{[32]}$ for spin-resolved ARPES experiments, which was recently used to map the band structure of topological insulators. ${ }^{[33-35]}$ In the following, recent activities involving femtosecond laser pulses are discussed in more detail.

\section{Time-resolved 2PPE Studies of Surface and Interface States}

Hexagonal boron nitride ( $h$-BN) monolayers on a $\mathrm{Ni}(111)$ surface $^{[36]}$ constitute an ideal model system to study electron transport at metal-insulator junctions. Whereas the occupied $\pi$ and $\sigma$ bands are well understood, much less is known about the unoccupied electronic states. Fig. 2(a) depicts the energy level scheme of the unoccupied states together with the free electron final states $(A, B, C)$ that were observed as features in the mono- and bichromatic 2PPE spectra given in Fig 2(b). ${ }^{[37]}$ The energetic position $0.65 \mathrm{eV}$ below $E_{\text {vac }}$ makes peak $A$ likely to be an image potential state. In a pump-probe experiment, only the intensity of peak $C$ was dependent on the delay between the red and blue laser pulses. More interestingly, the lifetimes obtained from the pump-probe (cross correlation) measurements of peak $C$ vary with the laser wavelength as shown in Fig. 2(c). As indicated, positive delays suggest a red pump and blue probe pulse, negative delays the opposite. It can therefore be concluded that two different excitation schemes via different intermediate states lead to the same final state that is responsible for the composite peak $C$. The energetic position of the intermediate state in the blue pumpred probe process coincides with the position of the intermediate state of peak $A$ and we can safely assign it to an image potential state, which is also in good agreement 


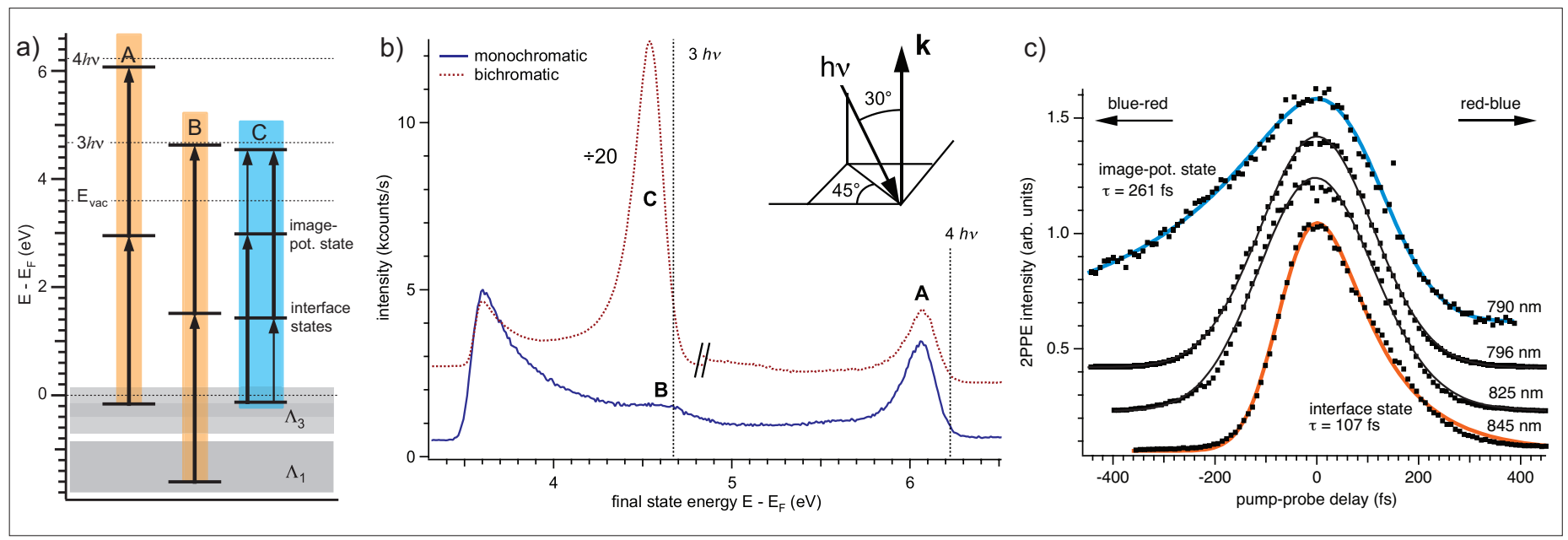

Fig. 2. (a) Energy level diagram deduced from experimental results. States observed in the 2PPE spectra are marked with horizontal lines. Monochromatic (orange) and bichromatic (blue) processes lead to different final states, short and long arrows indicate transitions at 796 and 398 $\mathrm{nm}$, respectively. (b) 2PPE spectra of the $h$-BN/Ni(111) interface excited with 796 and 398 nm laser pulses. The monochromatic spectrum was taken using blue pulses only. (c) 2PPE intensity of feature $\mathrm{C}$ in the photoelectron spectrum as a function of pump-probe delay at different wavelengths. Adapted from ref. [37].

with the measured long lifetime of $261 \mathrm{fs}$. Likewise the intermediate states of peak $B$ and the red pump-blue probe process of $C$ coincide and its energetic position $1.51 \mathrm{eV}$ above $E_{\mathrm{F}}$ identifies it as an interface state with a surprisingly long lifetime of $107 \mathrm{fs}$.

To further illustrate the depth of information that can be gained form multiphoton experiments, normal emission 2PPE $(h v=3.08 \mathrm{eV})$ spectra from $\mathrm{Cu}(111)$ are given in Fig. 3. ${ }^{[38]}$ A maximum intensity at laser polarization angles of $0^{\circ}$ and $180^{\circ}$ ( $p$-polarized) is indicative of a transition dipole moment normal to the surface. This is typical for a transition out of a Shockley surface state, since its main orbital character is $p$, where $z$ points out of the surface plane.

\section{Ultrafast Electron Transfer at the Diamondoid-SAM/Ag(111) Interface}

Diamondoid molecules are promising candidates for a large variety of applications where functionalized building blocks with nanometer dimensions are required. ${ }^{[39]}$ Both hydrogen-terminated bulk diamond and nano-structured diamond grown by chemical vapor deposition exhibit negative electron affinity (NEA). ${ }^{[40]}$ It has recently been demonstrated by means of a PES experiment that self-assembled monolayers (SAM) of [121] tetramantane-6-thiol on $\operatorname{Ag}(111)$ and $\mathrm{Au}(111)$ show similar NEA behavior. ${ }^{[41]}$ Yang et al. detected a sharp peak at the lowkinetic energy threshold of the emission spectrum, containing up to $68 \%$ of the total spectral weight. They also noticed the potential application of diamondoids as highly monochromatic electron emitters, which motivated us to investigate the emission process in more detail. In a 2PPE experiment, a sharp peak (FWHM $0.15 \mathrm{eV}$ ) at the low-en-

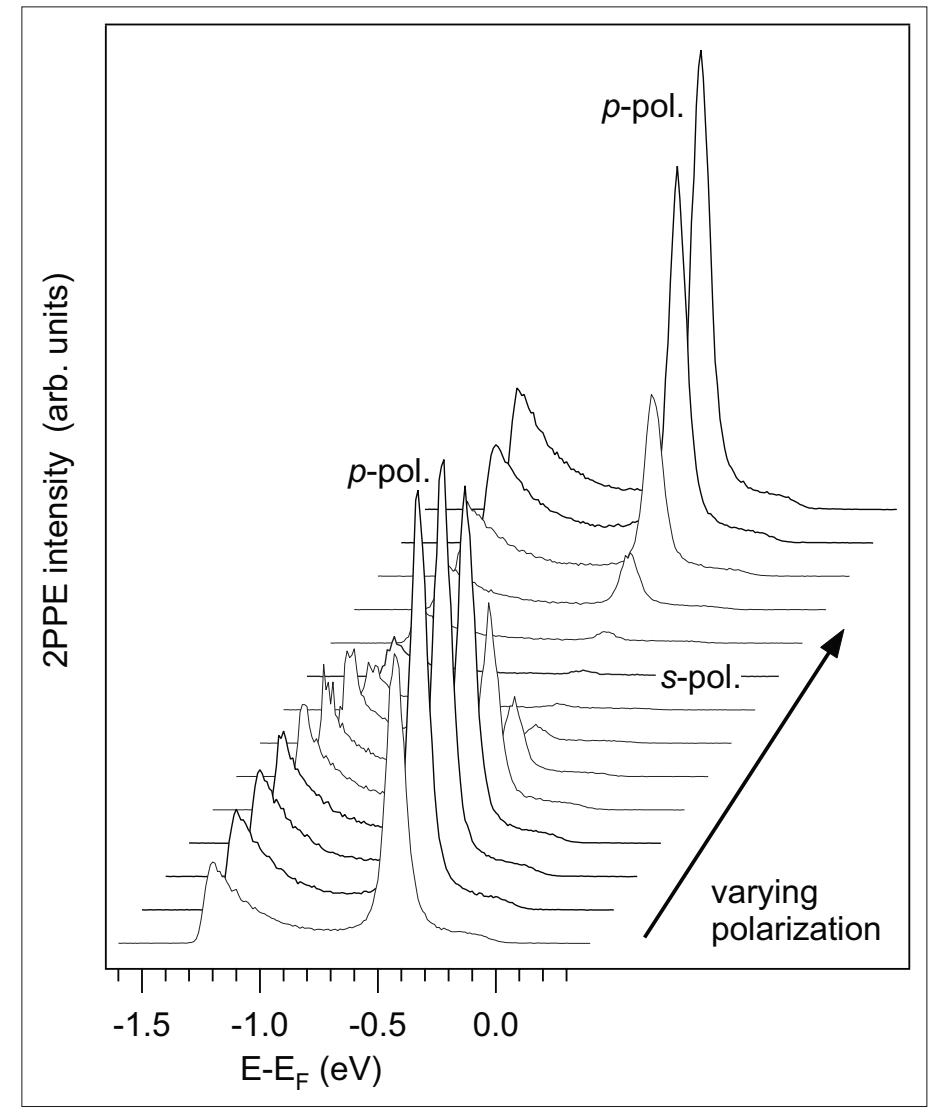

Fig. 3. Normal emission spectra from $\mathrm{Cu}(111)$ for varying orientation of the laser polarization. From ref. [38].

ergy cut-off with a spectral weight of about $80 \%$ was observed. ${ }^{[42]}$ It is assumed that highly excited electrons are transferred from the metal to the molecule and accumulate in the LUMO. The electrons are then emitted from the LUMO, which is located above the vacuum level as shown in Fig. 4. From our measurements, electron affinities of -0.21 $\mathrm{eV}$ for the $\mathrm{Ag}$ and $-0.57 \mathrm{eV}$ for the Au substrate could be derived. Attempts to clock the emission process by means of pumpprobe experiments and electron streaking were unsuccessful but we can provide an upper bound on the order of only a few femtoseconds. In addition to monochromaticity, diamondoids also provide a potential source of ultra-short electron pulses.

\section{Summary and Outlook}

A brief summary of photoelectron spectroscopy was provided and illustrated by several seminal contributions from other groups in the field as well as by examples from our own research. We hope we could provide 


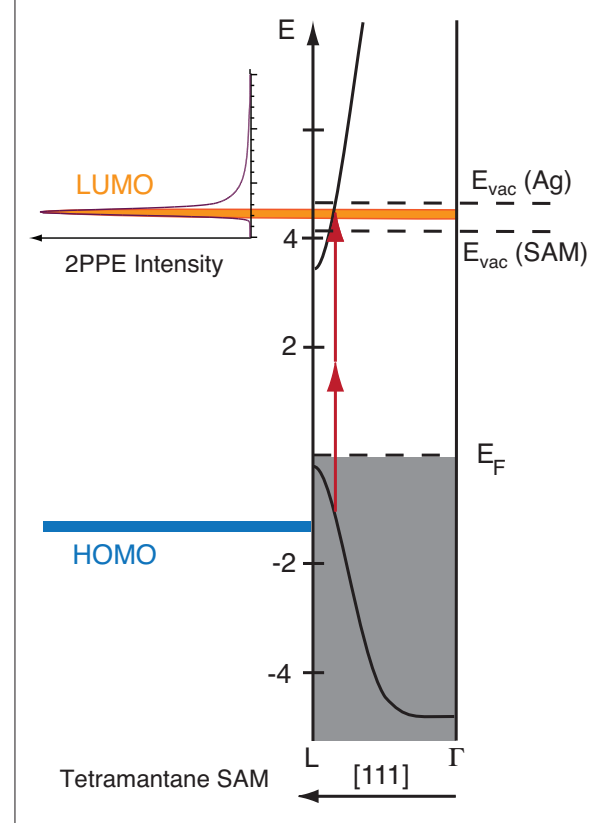

Fig. 4. $\mathrm{Ag}(111)$ substrate bandstructure and molecular orbitals in the [121]tetramantane-6thiol SAM. The two-photon threshold transition into the LUMO (orange line) is indicated with red arrows. Adapted from ref. [42].

a good overview of the potential power of PES, and in particular its time-resolved variant to study ultrafast processes at surfaces and interfaces. We are currently establishing several collaborations within NCCR MUST to push the temporal resolution into the attosecond domain, to use shaped pulses for the preparation of well-defined quantum states, and ultimately to build a time-resolved structural probe. For this purpose, we are building a new mobile photoelectron spectrometer, which can be used with the various light sources that are available at different laboratories within the network.

\section{Acknowledgements}

Financial support from the Swiss National Science Foundation (SNF) and the University of Zurich is gratefully acknowledged.

Received: February 28, 2011

[1] S. Hüfner, 'Photoelectron Spectroscopy', Springer, Berlin, 1995.

[2] H. Hertz, Ann. Physik 1887, 33, 983.

[3] A. Einstein, Ann. Physik 1905, 17, 132

[4] K. Siegbahn, Ark. Fys. 1954, 7, 86.

[5] C. Nordling, E. Sokolowsk, K. Siegbahn, Phys. Rev. 1957, 105, 1676.

[6] W. E. Spicer, Phys. Rev. 1958, 112, 114.

[7] M. P. Seah, W. A. Dench, Surf. Interface Anal. 1979, 1,2 .

[8] H.-L. Dai, W. Ho, 'Laser Spectroscopy and photochemistry on metal surfaces', World Scientific, Singapore, 1995.

[9] G. Böhm, K. Ulmer, Z. Phys. 1969, 228, 473.

[10] H. Petek, S. Ogawa, Prog. Surf. Sci. 1997, 56 , 239

[11] W. Ho, J. Phys. Chem. 1996, 100, 13050

[12] T. Hertel, E. Knoesel, M. Wolf, G. Ertl, Phys. Rev. Lett. 1996, 76, 535.

[13] S. Pawlik, M. Bauer, M. Aeschlimann, Surf. Sci. 1997, 377-379, 206.
[14] E. Knoesel, A. Hotzel, M. Wolf, Phys. Rev. $B$ 1998, 57,12812

[15] P. M. Echenique, J. B. Pendry, J. Phys. C 1978, $11,2065$.

[16] R. W. Schoenlein, J. G. Fujimoto, G. L. Eesley, T. W. Capehart, Phys. Rev. B 1991, 43, 4688.

[17] U. Höfer, I. L. Shumay, C. Reuss, U. Thomann, W. Wallauer, T. Fauster, Science 1997, 277, 1480.

[18] P. B. Corkum, F. Krausz, Nature Phys. 2007, 3, 381.

[19] M. Hentschel, R. Kienberger, C. Spielmann, G. A. Reider, N. Milosevic, T. Brabec, P. Corkum, U. Heinzmann, M. Drescher, F. Krausz, Nature 2001, 414, 509.

[20] C. N. Berglund, W. E. Spicer, Phys. Rev. 1964, 136, A1030.

[21] A. L. Cavalieri, N. Müller, T. Uphues, V. S. Yakovlev, A. Baltuška, B. Horvath, B. Schmidt, L. Blümel, R. Holzwarth, S. Hendel, M. Drescher, U. Kleineberg, P. M. Echenique, R. Kienberger, F. Krausz, U. Heinzmann, Nature 2007, 449, 1029.

[22] M. Schultze, M. Fieß, N. Karpowicz, J. Gagnon, M. Korbman, M. Hofstetter, S. Neppl, A. L. Cavalieri, Y. Komninos, T. Mercouris, C. A. Nicolaides, R. Pazourek, S. Nagele, J. Feist, J. Burgdörfer, A. M. Azzeer, R. Ernstorfer, R. Kienberger, U. Kleineberg, E. Goulielmakis, F. Krausz, V. S. Yakovlev, Science 2010, 328, 1658.

[23] T. Greber, O. Raetzo, T. J. Kreutz, P. Schwaller, W. Deichmann, E. Wetli, J. Osterwalder, Rev. Sci. Instr. 1997, 68, 4549.

[24] T. Greber, T. J. Kreutz, J. Osterwalder, Phys. Rev. Lett. 1997, 79, 4465.

[25] F. Baumberger, T. Greber, J. Osterwalder, Phys. Rev. B 2000, 62, 15431.

[26] F. Baumberger, T. Greber, B. Delley, J. Osterwalder, Phys. Rev. Lett. 2002, 88, 237601.

[27] F. Baumberger, W. Auwarter, T. Greber, J. Osterwalder, Science 2004, 306, 2221.

[28] T. Greber, J. Wider, E. Wetli, J. Osterwalder, Phys. Rev. Lett. 1998, 81, 1654.

[29] J. Wider, T. Greber, E. Wetli, T. J. Kreutz, P. Schwaller, J. Osterwalder, Surf. Sci. 1998, 417, 301.
[30] R. Fasel, A. Cossy, K.-H. Ernst, F. Baumberger, T. Greber, J. Osterwalder, J. Chem. Phys. 2001, 115,1020

[31] R. Fasel, J. Wider, C. Quitmann, K.-H. Ernst, T. Greber, Angew. Chem. Int. Ed. 2004, 43, 2853.

[32] M. Hoesch, T. Greber, V. Petrov, A. Muntwiler, M. Hengsberger, W. Auwarter, J. Osterwalder, J. Electron Spectrosc. Relat. Phenom. 2002, 124, 263.

[33] D. Hsieh, Y. Xia, D. Qian, L. Wray, J. H. Dil, F. Meier, J. Osterwalder, L. Patthey, J. G. Checkelsky, N. P. Ong, A. V. Fedorov, H. Lin, A. Bansil, D. Grauer, Y. S. Hor, R. J. Cava, M. Z. Hasan, Nature 2009, 460, 1101.

[34] D. Hsieh, Y. Xia, D. Qian, L. Wray, F. Meier, J. H. Dil, J. Osterwalder, L. Patthey, A. V. Fedorov, H. Lin, A. Bansil, D. Grauer, Y. S. Hor, R. J. Cava, M. Z. Hasan, Phys. Rev. Lett. 2009, 103, 146401

[35] D. Hsieh, Y. Xia, L. Wray, D. Qian, A. Pal, J. H. Dil, J. Osterwalder, F. Meier, G. Bihlmayer, C. L. Kane, Y. S. Hor, R. J. Cava, M. Z. Hasan, Science 2009, 323, 919.

[36] W. Auwarter, T. J. Kreutz, T. Greber, J. Osterwalder, Surf. Sci. 1999, 429, 229.

[37] M. Muntwiler, M. Hengsberger, A. Dolocan, H. Neff, T. Greber, J. Osterwalder, Phys. Rev. B 2007, 75, 075407.

[38] M. Hengsberger, F. Baumberger, H. J. Neff, T. Greber, J. Osterwalder, Phys. Rev. B 2008, 77, 085425.

[39] J. E. Dahl, S. G. Liu, R. M. K. Carlson, Science 2003, 299, 96.

[40] W. Zhu, G. P. Kochanski, S. Jin, Science 1998 $282,1471$.

[41] W. L. Yang, J. D. Fabbri, T. M. Willey, J. R. I. Lee, J. E. Dahl, R. M. K. Carlson, P. R. Schreiner, A. A. Fokin, B. A. Tkachenko, N. A. Fokina, W. Meevasana, N. Mannella, K. Tanaka, X. J. Zhou, T. van Buuren, M. A. Kelly, Z. Hussain, N. A. Melosh, Z.-X. Shen, Science 2007, 316, 1460

[42] S. Roth, D. Leuenberger, J. Osterwalder, J. E. Dahl, R. M. K. Carlson, B. A. Tkachenko, A. A. Fokin, P. R. Schreiner, M. Hengsberger, Chem. Phys. Lett. 2010, 495, 102. 